\title{
A rapid automatic analyzer and its methodology for effective bentonite content based on image recognition technology
}

\author{
*Wei Long ${ }^{1,2}$, Lu Xia ${ }^{1,2}$, and Xiao-lu Wang ${ }^{1,2}$ \\ 1. School of Materials and Chemical Engineering, Hubei University of Technology, Wuhan 430068, China; \\ 2. Hubei Provincial Key Laboratory of Green Materials for Light Industry, Wuhan 430068, China
}

\begin{abstract}
Fast and accurate determination of effective bentonite content in used clay bonded sand is very important for selecting the correct mixing ratio and mixing process to obtain high-performance molding sand. Currently, the effective bentonite content is determined by testing the ethylene blue absorbed in used clay bonded sand, which is usually a manual operation with some disadvantages including complicated process, long testing time and low accuracy. A rapid automatic analyzer of the effective bentonite content in used clay bonded sand was developed based on image recognition technology. The instrument consists of auto stirring, auto liquid removal, auto titration, step-rotation and image acquisition components, and processor. The principle of the image recognition method is first to decompose the color images into three-channel gray images based on the photosensitive degree difference of the light blue and dark blue in the three channels of red, green and blue, then to make the gray values subtraction calculation and gray level transformation of the gray images, and finally, to extract the outer circle light blue halo and the inner circle blue spot and calculate their area ratio. The titration process can be judged to reach the end-point while the area ratio is higher than the setting value.
\end{abstract}

Key words: used clay bonded sand; bentonite; ethylene blue absorbed; image recognition; automatic analyzer

CLC numbers: TG221

Document code: A

Article ID: 1672-6421(2016 05-322-05

$\mathrm{I}^{\mathrm{t}}$

$t$ is one of the most critical issues to ensure stable quality of clay molding sand in mass-production of castings ${ }^{[1-2]}$. Real-time and rapid detection of the molding sand performances is the premise for mixing high performance molding sand and improving casting quality ${ }^{[3,4]}$. There are many factors that affect the performances of green molding sand. One of the important factors is the effective bentonite content in the used clay bonded sand. To quickly and accurately determine the effective bentonite content in used clay sand is the basis of determining the correct mixing ratio and mixing process of molding sand with highperformances.

At present, the main determination method for effective bentonite content in used clay sand is manual titration on the moulding sand production line ${ }^{[5]}$. The effective bentonite content is determined by testing the ethylene

\section{*Wei Long}

Male, born in 1982, lecturer. Research interest: quality control of clay bonded molding sand.

E-mail: maillong1982@126.com

Received: 2015-12-24; Accepted: 2016-08-30

blue absorbed in used clay bonded sand, and the basis of judging the titration end-point is the light blue halo being presented on the outer circle of the spot on the neutral quantitative filter paper. The complex and time consuming test process often causes measurement errors. Based on manual titration, the SND-type methylene blue clay tester was developed in China ${ }^{[5]}$. The tester could achieve the auto-titration through manually controlling the pressure of a balloon, which, however, is a timeconsuming, complex process with low recognition precision by the naked eye.

R. C. Ren ${ }^{[6]}$, etc. developed an automatic analysis detector based on the bentonite ethylene blue absorption. The detector consists of three parts: auto-titration, sampling system, and color recognition. The methylene blue titer quantity is controlled by a man-machine dialogue system. A given color calibration is used as the color recognition system of detector, and the color sensor is used to detect the reseda halo appearing in the titration spot under the fluorescence illumination. Comparing the halo with the given color calibration, the titration endpoint could be determined. The five stepper motors are used in the detector, resulting in a relatively complex 
operation process. The filter-rods dipping in solution method is used in the liquid removal process, but the droplet could be a previous drop, leading to an empty drop when the filter-rods are mechanically driven by stepper motors, which would result in repeated titration. In the later data processing, the effective bentonite content is calculated through manually balancing the standard titration curve of bentonite, thus the data processing is more complex.

In the present study, a rapid automatic analyzer for effective determination of bentonite content in clay bonded sand was developed by using computer control technology and image recognition technology. It uses the photosensitive degree difference of the light blue and dark blue in the three color channels of red, green and blue, by firstly decomposing the color images into three-channel gray images, then making the gray value subtraction calculation and gray level transformation of the gray images, finally extracting the outer circle light blue halo and the inner circle blue spot to calculate their area ratio. When the area ratio is higher than the set value, the end-point is reached, and the titration process is ended. The instrument can accurately and quickly determine the effective bentonite content in used clay bonded sand, and therefore, it can be used to adjust the mixing sand ratio in time in the clay molding sand production line, and reduce casting defects.

\section{Construction of rapid automatic analyzer for effective bentonite content based on image recognition}

\subsection{Hardware construction}

The structure sketch of the rapid automatic analyzer for effective bentonite content in used clay bonded sand is shown in Fig. 1. The instrument consists of a stirring component (5 in Fig.1), liquid removal parts $(3,4)$, titration units $(7,8)$, step-rotation parts $(11,12)$, image acquisition units $(9,10)$ and processor $(13)$. The operation of stirring component, liquid removal part, titration unit, step-rotation and image acquisition parts are controlled by the program control module and the image processing module in the processor. After the titration process the image acquisition unit collects the color spot image, and then it transfers the image to the processor. Finally, the processor manages the color spot image data to judge the titration end-point.

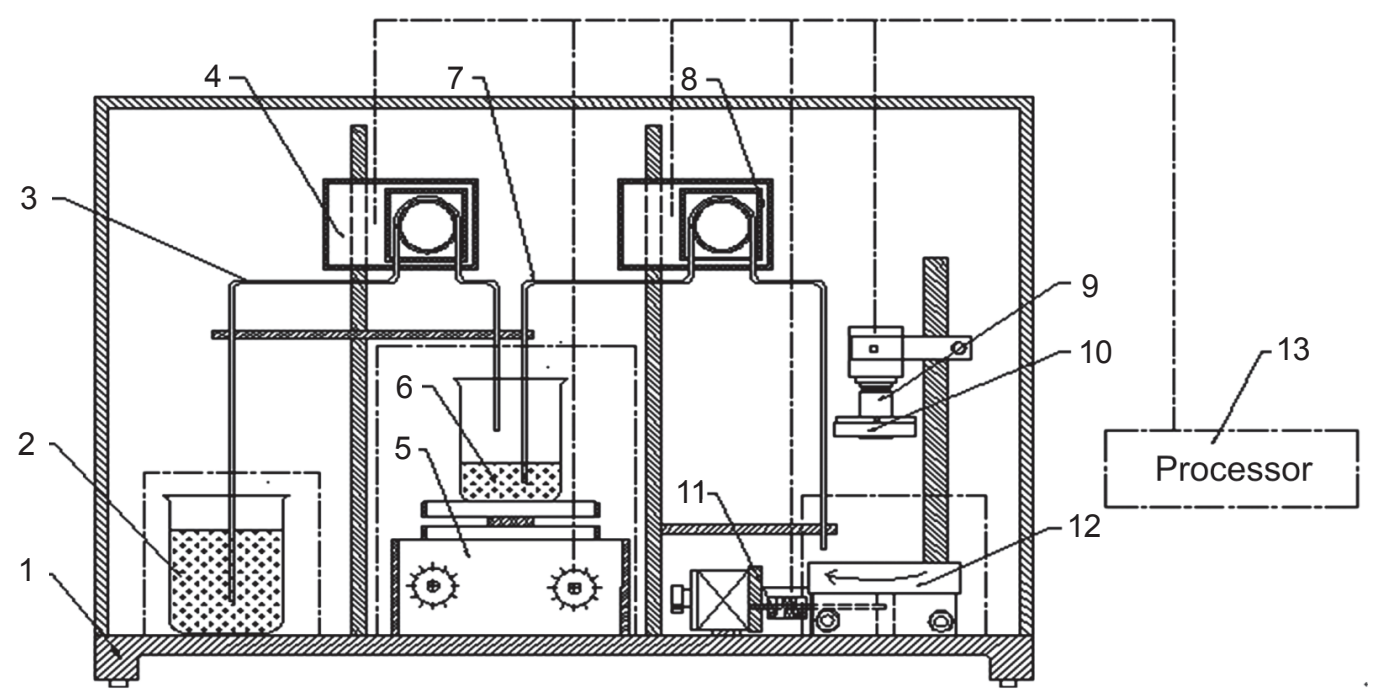

1- shell; 2- reagent container; 3- pipette; 4- liquid removing peristaltic pump; 5- stirring unit; 6- specimen container; 7- buret; 8- titration peristaltic pump; 9- digital industry camera; 10- circular illumination; 11- step motor; 12- rotating platform; 13-processor

Fig. 1: Structure sketch of rapid automatic analyzer of effective bentonite content in used clay green sand

\subsection{Titration process of rapid automatic analyzer}

The titration process of the auto-fast determination instrument for effective bentonite content in the used clay bonded sand includes the following six steps:

(1) The specimen container with the mixed solutions of used clay bonded sand samples is set on the stirring unit and heated to disperse the solutions;

(2) After the mixed solution is cooled to room temperature, the methylene blue solution is removed quantitatively from the reagent container to the specimen container by the liqid removing component, at the same time, the stirring unit is driven to stir the solution;

(3) The mixed solution in the specimen container is titrated on the filter paper on the rotating platform;

(4) After collecting a drop of mixed solution, the platform is rotated a certain angle by step motor, then the underside titration spot is collected by the image acquisition unit, and then the spot image is transferred to the processor;

(5) The drop spot image data is treated by the processor;

(6) The titration end-point is determined by repeating above steps (2) to (5). 


\section{Realization of auto-liquid removal and titration process}

The auto-quantitative liquid removal process and the titration process are realized by controlling the liquid removal time and speed. The liquid removal time is determined by the rotational speed $(N)$ of the peristaltic pump and the inner diameter $(R)$ of the pipette. The liquid removal and titration process includes three stages:

(1) The pipette liquid-filling stage. The pipette is completely filled with methylene blue solution under the rotational speed $N_{1}$ of the peristaltic pump at the beginning of liquid movement. Because the pipette length and the pump rotational speed are constant values, the liquid-filling time of pipette is considered as a constant value, $T_{1}$, and the liquid-filling volume of methylene blue solution is set as $V_{1}$.

(2) The fast titration stage. The methylene blue solution is titrated to the mixed solution of sand and water under the fast rotational speed $N_{2}$ of the peristaltic pump. The fast titration time is set as $T_{2}$ and the titration volume of methylene blue solution as $V_{2}$. The volume $V_{2}$ is about $90 \%$ of total titration volume.

(3) The slow titration stage. When titration closes to the endpoint, the rotational speed of the peristaltic pump is reduced to $N_{3}$, and the $0.1 \mathrm{ml}$ methylene blue solution is titrated to the specimen container every cycle till reaching the titration endpoint. The slow titration time is set as $T_{3}$ and the total titration volume of the stage (3) as $V_{3}$.

The total titration time, $T$, is the sum of $T_{1}, T_{2}$ and $T_{3}$. The total titration volume of methylene blue solution is $V$, that is, the sum of $V_{2}$ and $V_{3}$. The pipette liquid-filled volume $V_{1}$ is reserved in pipette.

In the automatic liquid removal and titration process, the peristaltic pump rotational speed $N_{1}, N_{2}$ and $N_{3}$ in each stage could be input to the program control module of the processor as the constant parameters, so that the module can control the liquid removal and titration process automatically.

\section{Image recognition principle and process}

Image recognition is the key technology of the rapid automatic analyzer for determination of the effective bentonite content

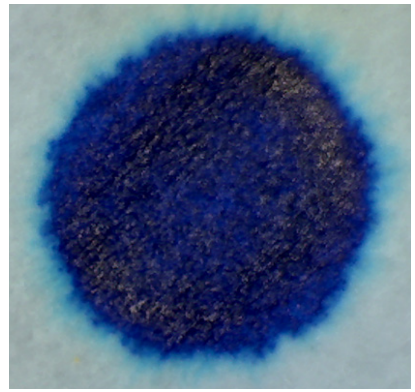

(a) Original color image

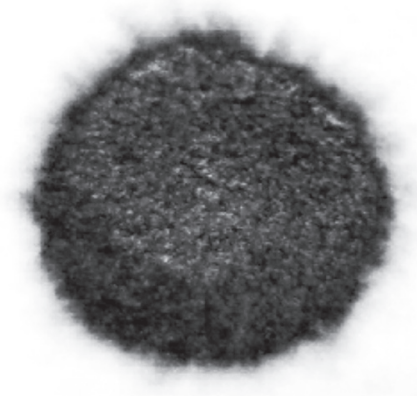

(b) Red channel image in used clay bonded sand. In the slow titration stage, after 0.1 $\mathrm{ml}$ methylene blue solution is titrated for each cycle, a drop of uniform mixed solution is titrated to the filter paper on the rotating platform, and the titration spot image is obtained. Then the spot image is transferred into the processor to get image data. Finally, the image data is compared with the setting value in the processor. After multiple cycles of testing, the titration end-point is achieved.

\subsection{Image recognition principle}

The titration spot image is a color image collected by the image acquisition unit. The color image has higher pixel density than the black-and-white image, so it could accurately recover the primitive titration spot on the filter paper, and improve the image recognition accuracy.

The image recognition principle is to use the photosensitive degree difference of the light blue and dark blue in the three color channels of red, green and blue to identify the spot image. First, the color spot image is decomposed into a three-channel gray image, then the gray value subtraction operation and gray level transformation are made, and finally, the outer circle light blue halo and inner circle blue core in spot image are extracted separately and their area ratio are calculated. When the area ratio is higher than the set value, the titration process is considered to reach the end-point, and the testing system would calculate the ethylene blue adsorbed value and the effective bentonite content of the used clay bonded sand automatically.

\subsection{Realization of image recognition process}

\subsubsection{R/G/B three-channel decomposition of color image}

The RGB color mode is a kind of industrial standard color. All types of colors are obtained by changing their red (R), green (G) and blue (B) color channels and stacking above three color channels ${ }^{[7]}$. When the collected spot image data are treated by the processor, it is decomposed into the RGB three-channel gray image at first. Figure 2 shows the original color image and the three-channel decomposition images when the titration reached the end-point.

Figure 2(a) shows the original image of titration spot. Figure 2(b) is the gray image with red channel, which can display completely the image color layer changes. Figure 2(c) and 2(d) are respectively the gray images with green channel and blue channel, in which the inner circle blue spot could be displayed but the outer

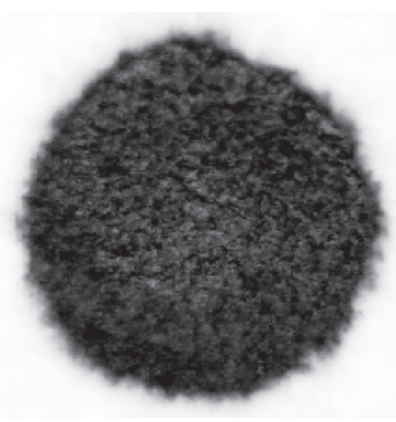

(c) Green channel image

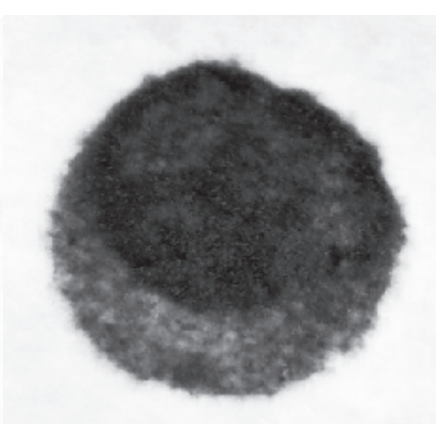

(d) Blue channel image

Fig. 2: Original color image and three-channel decomposition images 
circle light blue halo could not virtually be displayed. Comparing Fig. 2(c) with Fig. 2(d), the color changes of the inner circle blue spot in the original image is displayed not obvious in Fig. 2(c), but it could be clearly seen in Fig. 2(d), so the difference of the green channel gray image Fig. 2(c) and the blue channel gray image Fig. 2(d) could be used in the channel subtraction operation to remove the desultory points and to obtain the full outer circle light blue halo.

\subsubsection{RGB three-channel subtraction operation}

On the basis of the different characteristics of spot images in red, green and blue channels, a complete light blue halo could be obtained by the channel subtraction operation and gray level transformation ${ }^{[7-8]}$. The three-channel subtraction operation diagrams are shown in Fig. 3. Gray level transformation function is a linear function ${ }^{[9]}$, as described in equation (1):

$$
f_{1}=a \times f_{0}+b
$$

where the parameters $a$ and $b$ are the constants, $f_{0}$ and $f_{1}$ are the gray values of one image pixel point before and after the gray level being transformed, respectively.

Figure 3 shows the RGB three-channel subtraction operation results. Figure 3(b) is obtained by (R-G) channel subtraction in Fig. 2. It can be seen that some desultory points exist on the Fig. 3(b) after the (R-G) channel subtraction and gray level transforming. The complete halo displayed in Fig. 3(c) is obtained after the $[(\mathrm{R}-\mathrm{G})-\mathrm{B}]$ channel subtraction and gray level transformation, and the desultory points disappear.
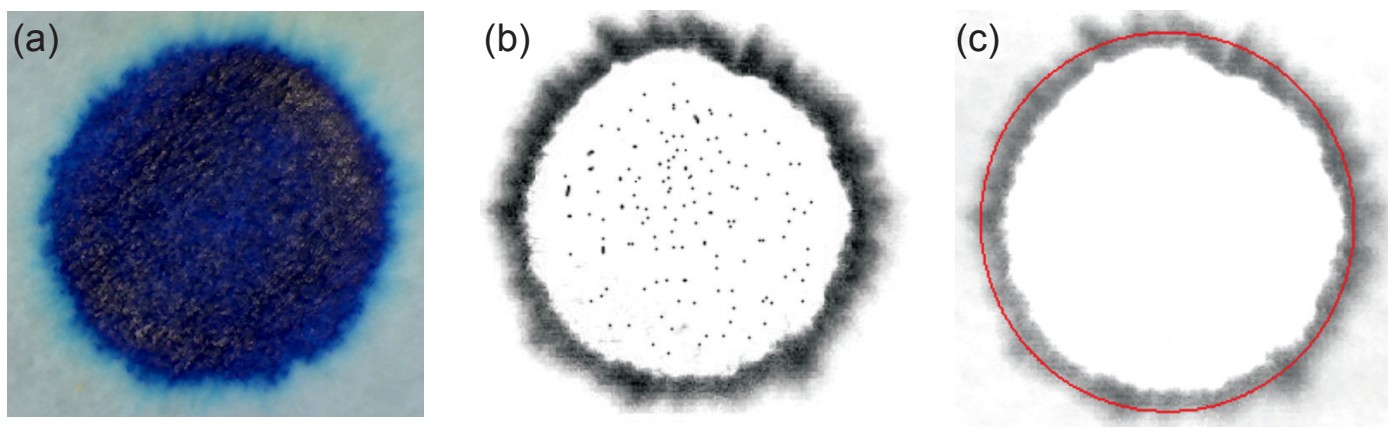

Fig. 3: Flowchart of RGB three-channel subtraction operation

\subsubsection{Halo area calculation and transition point definition}

Because the image is made up of many pixel points, the light blue halo area and the center blue spot area can be obtained by calculating the pixel point numbers in different areas when the area of one pixel point is constant. Supposing the actual area of one pixel is $f(d)$ under different resolution ratio, then the light blue halo area $A_{1}$ is:

$$
A_{1}=f(d) \cdot \int S_{1}(d)
$$

where the parameter $d$ is the digital camera resolution ratio, $\int S_{1}(d)$ is the total number of pixels which are contained in the halo image region.

In the same way, the center blue spot image is obtained by making the eight neighborhoods image-filling ${ }^{[10-12]}$, the center blue spot area $A_{2}$ is:

$$
A_{2}=f(d) \cdot \int S_{2}(d)
$$

where the $\int S_{2}(d)$ is the total number of pixels contained in the center blue spot region.

Experimental results show that the $A_{1} / A_{2}$ value could be used to decide the titration end-point.

The light blue halo is the production of methylene blue solution reaction with the medium-speed quantitative filter paper. The effective bentonite content in used clay bonded sand is determined by the ethylene blue absorption amount of the used clay bonded sand solution. At the beginning of titration, the light blue halo does not appear on the filter paper although the methylene blue solution has been added into the used clay bonded sand solution, but an inconspicuous light blue halo would appear on the filter paper as the methylene blue solution addition amount increases to near the titration end-point, and an obvious light blue halo would appear on the filter paper when the excessive methylene blue solution is added. Therefore, it is important to define the transition-point of light blue halo, which is also the titration end-point. The transition-point is defined as that the ratio of the light blue halo area to the center blue spot area titrated on filter paper shows an obvious transition, and the light blue halo can be seen with the naked eye. A lot of experimental data has shown that the transition point can be defined as the ratio at $15 \%$.

Figure 4 shows the curve of the effective bentonite content of used clay bonded sand vs. titration area ratio. It can be seen that the area ratio of the light blue halo with the center blue spot has an obvious transition when the titer increases from $74 \mathrm{ml}$

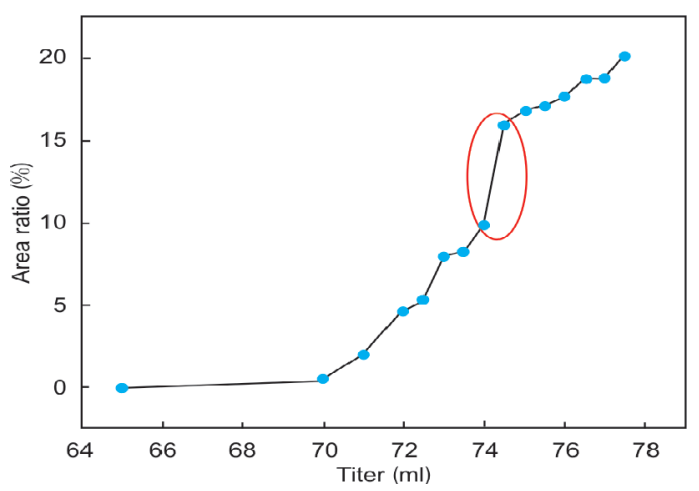

Fig. 4: Curve of titration area ratio vs. effective bentonite content 
to $74.5 \mathrm{ml}$. Figure 5 shows the change of spot images before $(74 \mathrm{ml})$ and after $(74.5 \mathrm{ml})$ titration end-point. An obvious light blue halo appeared on the outer circle of the spot in Fig. 5(b) as compared with that in Fig. 5(a).
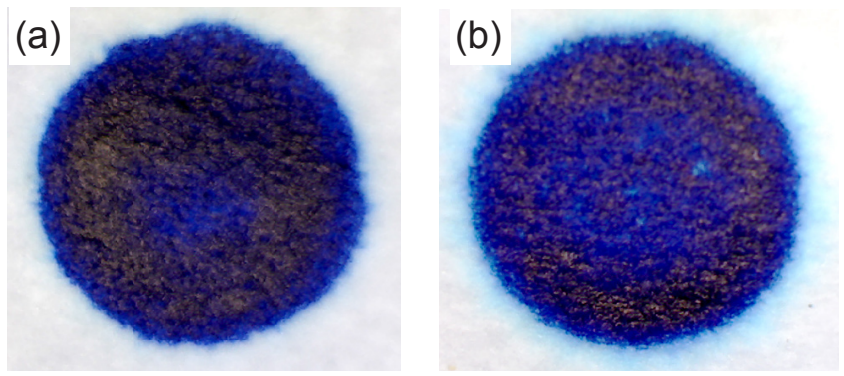

Fig. 5: Change of spot images before and after titration end-point

\section{Applications}

To validate the rapid automatic analyzer, the ethylene blue adsorbed amount of the used sand in the production of a foundry shop was tested using both a manual testing method and the rapid automatic analyzer, and then the effective bentonite contents were calculated. The results are shown in Fig. 6.

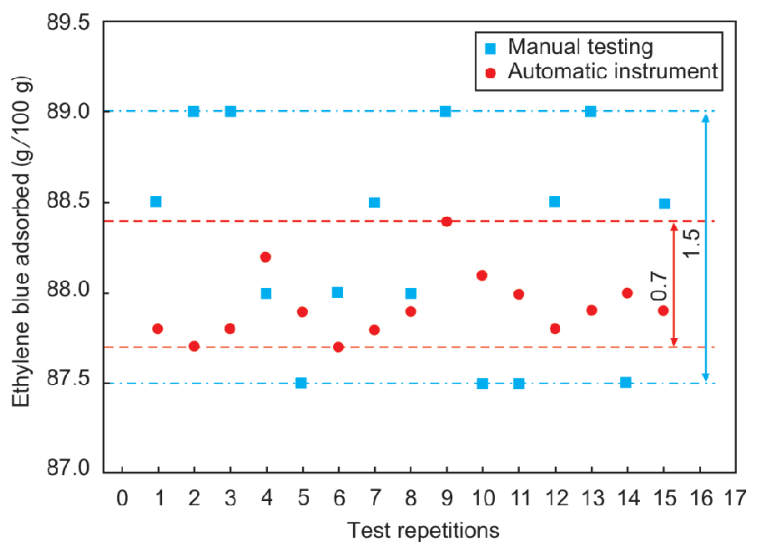

Fig. 6: Fluctuation range comparison of test results by manual method and rapid automatic analyzer for one batch used sand ethylene blue adsorbed

As shown in Fig. 6, the testing results by the rapid automatic analyzer have a smaller fluctuation range (about half) as compared to those by manual titration, indicating that the autofast determination instrument is more stable. Assuming that the average value of the effective bentonite ethylene blue adsorbed in used sand by manual test is the true value in theory, the standard deviations of manual titration and automatic titration are calculated to be 0.59 and 0.43 , respectively. The standard deviation of the rapid automatic analyzer is less than the manual titration, indicating that the test results from the rapid automatic analyzer are closer to the true value, and therefore, have a higher reliability.

\section{Conclusions}

(1) The rapid automatic analyzer and its methodology for determining effective bentonite content are based on image recognition technology, which overcomes the disadvantages of the manual operation such as time consumption and process complexity, and presents some advantages including simple operation, automation and computerization.

(2) The components used in the instrument are mature products on the market. The light blue halo area and the center blue spot area can be recognized and calculated automatically using advanced computer image processing technology.

(3) Test results show that the new instrument and method of determining the effective bentonite content in the used clay bonded sand is more stable, precise and reliable as compared with the manual method. It should be suitable for determining the effective bentonite content in all kinds of clay bonded sands.

\section{References}

[1] Zhao Shucheng. Problem and measures for improvement on quality of molding sand. China Foundry Machinery and Technology, 2000(1): 34-38. (In Chinese)

[2] Long Wei, Fan Zitian, Zou Wei, et al. The effect of ambient temperature and used sand temperature on clay mould sand performances. Foundry, 2007(1): 75-78. (In Chinese)

[3] Li Dayong. Study on the technology of testing available clay moisture in green sand rapidly with new method. In: Proceedings of the 4th Asian Foundry Congress, Australia, 1996.

[4] Hattai T, et al. Influence of preparation method of test specimen green sand measurements. In: Proceedings of the 65th World Foundry Congress, Korea: Gyeongju, 2002, 859-867.

[5] Foundry Institution of Chinese Mechanical Engineering Society. Casting Manual (4), Beijing: China Machine Press, 2002. (In Chinese)

[6] Ren R C, Xue Z G, Yang L, et al. Auto- analyses instrument and its method of the bentonite ethylene blue absorbed. China patent: CN 101493451A, 2009-7-29.

[7] Steger C, Ulrich M, Wiedemann C. Machine Vision Algorithms and Applications. Beijing: Tsinghua University Press, 2004.

[8] Luo Wencun. A New Image Segmentation Approach by Integration of Thresholding and Region Growing. Modern Computer, 2001(5): 43-45. (In Chinese)

[9] Jiang Zhongcheng, Zhou Chunlai, Ji Yunyi. Segmentation and Restoration of Bar Image with Complex Background. Micro Computer Information, 2007, 23(1-3): 291-293. (In Chinese)

[10] Hans Luijendijk .Automatic threshold selection using histograms based on the count of 4 -connected regions. Pattern Recognition Letters, 1991, 12(4): 219-228.

[11] Wang Lisheng, Bai Jing. Threshold selection by clustering gray levels of boundary. Pattern Recognition Letters, 2003, 24(12): 1983-1999.

[12] Sun Jiaguang. Computer Graphics (The third edition). Beijing: Tsinghua University Press, 1998. (In Chinese) 